\title{
Qualitätssicherung als Governanceproblem?
}

\author{
Gunnar Folke Schuppert*
}

Zugleich eine Besprechung von Franz Reimer, Qualitätssicherung - Grundlagen eines Dienstleistungsverwaltungsrechts, Baden-Baden (Nomos) 2010, 528 S., gebunden 119,- $€$, ISBN 978-3-8329-4726-2

A. Hochschullehrer als Qualitätsprüfer. . 334

B. Fünf für das Problem der Qualitätssicherung zentrale Kontextdiskurse..... 336

I Der „audit society“-Diskurs........ 336

1. Legitimation der EvaluationsMandarine................... 337

2. Beobachtungsstrukturen als Governancestrukturen.......... 338

3. Zur Problematik von Qualitätsindikatoren ..................... 339

4. Qualitätsbewertung als Governance von und durch Wissen.......................... 340

II. Der „Zur Rolle des Staates“-Diskurs
III. Der Ökonomisierungs-Diskurs ..... 342

IV. Der „Das Gesetz bekommt Gesellschaft"-Diskurs ..................... 344

V. Der Governance-Diskurs ............ 345

C. Drei Beispiele für qualitätssichernde Governanceregime ................. 347

I. Selbststeuerung durch peer review . 347

II. Akkreditierung im Hochschulbereich oder Steuerung durch Organisation ............................... 349

III. Qualitätssicherung durch Contract Governance....................... 351

D. Zusammenfassende Würdigung...... 353

Die hier zu besprechende Habilitationsschrift von Franz Reimer, die trotz ihres etwas irreführenden Untertitels nicht von den Grundlagen eines Dienstleistungsverwaltungsrechts, sondern von den Strukturen eines Qualitätssicherungsrechts handelt, beginnt mit dem schönen Satz „Qualität ist ein unwiderstehliches Argument“ und erklärt wenige Sätze später den Qualitätsbegriff zu einem Schlüsselbegriff einer heraufziehenden „Dritten Phase des öffentlichen Rechts in Deutschland“. Angesichts dieser argumentativen Wucht fragt man sich unwillkürlich, ob man selbst mit diesem Schlüsselbegriff bereits zu tun hatte oder sich etwa selbst schon im Geschäft der Qualitätssicherung betätigt hat. Wie wohl die meisten anderen Kollegen aus der „scientific community“ der Staatsrechtslehrer kann ich diese Frage bejahen und es mag daher erlaubt sein, vor der eigentlichen Auseinandersetzung mit der Reimerschen Arbeit mit einigen, meines Erachtens durchaus verallgemeinerbaren persönlichen Erfahrungen des Rezensenten als Qualitätsprüfer zu beginnen.

\section{A. Hochschullehrer als Qualitätsprüfer}

Erste Erfahrungen als Qualitätsprüfer konnte ich sammeln, als ich Ende der neunziger Jahre als Mitglied eines Gutachterteams der DFG über den Antrag auf Einrichtung und Finanzierung eines Sonderforschungsbereichs an der Universität Heidel-

* Prof. Dr. Gunnar Folke Schuppert war Inhaber des Lehrstuhls für Staats- und Verwaltungswissenschaft, insbesondere Staats- und Verwaltungsrecht, an der Humboldt-Universität zu Berlin und ist Inhaber einer Forschungsprofessur für „Neue Formen von Governance“ sowie Geschäftsführender Leiter des „Rule of Law Centers“ des Wissenschaftszentrum Berlin für Sozialforschung. 
berg (Politische Kultur in der Bundesrepublik Deutschland seit 1949) mit zu entscheiden hatte. Die hohen Qualitätshürden konnten in diesem Falle nicht übersprungen werden und das negative Urteil wurde von den Mitarbeitern der Deutschen Forschungsgemeinschaft professionell und geräuschlos exekutiert. Eine zweite permanent sprudelnde Erfahrungsquelle besteht in der Begutachtung von Manuskripten für solche die Methode des „peer review“ praktizierenden Zeitschriften wie etwa das Flaggschiff der Politikwissenschaft, die Politische Vierteljahresschrift (PVS). Ein dritter Einblick in das Geschäft der Qualitätssicherung wurde mir erst kürzlich als Mitglied einer Gutachtergruppe einer Akkreditierungsagentur eröffnet, die die Aufgabe hatte, zwei geplante Masterstudiengänge der Hochschule für Verwaltungswissenschaften in Speyer zu bewerten.

Diese Beispiele haben wir mit Bedacht an den Anfang gestellt, weil man aus ihnen eine erste zentrale Erkenntnis mitnehmen kann: Als Qualitätsprüfer ist man in aller Regel nicht als individueller Restaurant-Tester unterwegs, ${ }^{1}$ sondern ist eingebettet in ein der Qualitätssicherung dienendes Verfahren, das in bestimmten qualitätssichernden Strukturen abläuft und meistens von einer dazu berufenen Organisation „veranstaltet“ wird. Es geht also - dies ist der erste und wichtige Punkt, auf den noch zurückzukommen sein wird - eigentlich immer um Qualitätssicherungsregime, ${ }^{2}$ die ihrerseits Gegenstand einer Qualitätskontrolle sein können und es in der Regel auch sind.

Aber nun zu der Arbeit von Franz Reimer selbst. Es handelt sich bei ihr um eine anspruchsvolle und qualitätsbewusste Schrift zum Thema „Qualitätssicherung “, und zwar - als juristische Habilitationsschrift - vor allem zum Thema einer Qualitätssicherung durch und nach Maßgabe des Rechts. Dieses Thema wird - wie bei deutschen Habilitationsschriften üblich - von allen denkbaren Perspektiven aus in den Blick genommen und in dieser verwirrenden Vielfalt der Aspekte kann auch der Verfasser der „genretypischen “ Versuchung nicht widerstehen, bisweilen ins Enzyklopädische abzugleiten: dies ist deshalb schade, weil die zentralen Botschaften des Verfassers dadurch nicht immer deutlich genug hervortreten.

Wir haben uns daher entschlossen, statt der gängigen Rezensionsmethode, den Argumentationsgang der Arbeit nachzuzeichnen, eine andere zu wählen und das Problem der Qualitätssicherung in fünf unseres Erachtens nach zentrale Diskurse einzubetten, ohne die das Unterfangen der Entwicklung von Grundzügen eines Qualitätssicherungsrechts schwerlich gelingen kann; diese Diskurse scheinen uns der rich-

1 Zu den Spezifika der Evaluation von Restaurants siehe Manfred Kohnke, Von Sternen und Kochmützen. Evaluationen in der Haute Cuisine, in: Hildegard Matthies/Dagmar Simon (Hrsg.), Wissenschaft unter Beobachtung. Effekte und Defekte von Evaluationen, Leviathan-Sonderheft 24/2007, S. $347 \mathrm{ff}$.

2 Stefan Hornbostel, Neue Evaluationsregime? Von der Inquisition zur Evaluation, in: Matthies/Simon (Fn. 1), S. $58 \mathrm{ff}$. 
tige Ort zu sein, um dann gezielt und fokussiert die jeweiligen Überlegungen von Reimer einzubeziehen.

\section{B. Fünf für das Problem der Qualitätssicherung zentrale Kontextdiskurse}

\section{Der „audit society“-Diskurs}

Mit dieser inzwischen klassischen Formulierung von Michael Power ${ }^{3}$ soll der Befund auf den Begriff gebracht werden, dass wir in einer Gesellschaft leben, in der alles, aber auch wirklich alles beobachtet wird, und zwar nicht nur beobachtet, sondern als Ergebnis der Beobachtung auch evaluiert und in einer Rangliste platziert. ${ }^{4}$ Unser Lieblingsbeispiel ist die Messung von Lebensqualität durch den „Happy Planet Index“ der „New Economics Foundation “; 5 in diesem Standortwettbewerb um das Gemeinwesen mit der höchsten Lebensqualität hat die Inselgruppe Vanuatu den ersten Platz belegt, ein Ergebnis, das Richard David Precht in seinem ebenso tiefgründigen wie kurzweiligen Buch mit dem schönen Titel „Wer bin ich und wenn ja, wie viele? " 6 wie folgt kommentiert:

„Glaubt man dem Happy Planet Index, den die New Economics Foundation im Sommer 2006 veröffentlichte, dann ist Vanuatu das glücklichste Land der Welt. Vanua-was? Ja, das Land gibt es wirklich; ein vergleichsweise wenig bekannter Inselstaat im Südpazifik, den Älteren von uns möglicherweise noch als ,Neue Hebriden' aus dem Diercke-Schulatlas bekannt. Gefragt wurde nach den Erwartungen an das Leben, nach der allgemeinen Zufriedenheit und nach dem Verhältnis der Menschen zu ibrer Umwelt. Die optimale artgerechte Haltung für den Menschen wäre demnach ein Leben auf einer Vulkaninsel mit etwa 17 Einwohnern pro Quadratkilometer; ein mildes Klima mit viel Sonne und üppiger Vegetation; ein religiöser Mix aus Naturreligionen mit Protestanten, Anglikanern, Katholiken und Adventisten; bescheidene, aber ehrliche Arbeitsverhältnisse mit vielen Selbständigen; eine parlamentarische Demokratie mit einem starken Premierminister und einem schwachen Präsidenten und das britische Rechtssystem. Doch ganz so genau hatten es die Urheber der Studie, darunter auch die Umweltorganisation Friends of the Earth, gar nicht wissen wollen. Ihr eigentliches Ziel war herauszufinden, wie stark der Mensch in die Natur eingreifen und seine Umwelt schädigen muss, um Bedingungen zu schaffen, die ihm zum Glück verhelfen. Und die Antwort mit dem Sieger Vanuatu an der Spitze lautet: eher wenig!"

3 Michael Power, The Audit Society. Rituals of Verification, Oxford 1977.

4 Überblick mit weiteren Nachweisen bei Gunnar Folke Schuppert, Staat als Prozeß. Eine staatstheoretische Skizze in sieben Aufzügen, Frankfurt am Main/New York 2010, S. 89 ff.

5 New Economics Foundation, About us, 2006; abrufbar unter (http://happyplanetindex.org/).

6 München 2007, S. 347. 
Aber nicht nur Lebensqualität wird gemessen; dies könnte man ja noch als nicht so ernst zu nehmende Spielart einer um sich greifenden „Evaluitis“7 beiseite lassen. Gerated und gerankt wird wirklich alles: die Pannenanfälligkeit von Autos durch den nimmermüden ADAC, die Qualität von Hochschulen in den sog. Hochschulrankings, über die FOCUS und SPIEGEL gerne berichten, aber auch - und jetzt wird es auch politisch immer folgenreicher - die Kreditwürdigkeit von Staaten und Volkswirtschaften durch die großen Ratingagenturen - jüngste Beispiele sind die Herabstufungen von Portugal und Spanien - sowie die Leistungsfähigkeit und demokratische Qualität von Regierungen und Regierungssystemen.

Wenn sich dies aber so verhält, dass Qualitätsmessung und Qualitätssicherung ubiquitäre Phänomene sind, dann sollte man meinen, dass gemeinsame Strukturprobleme benennbar sind, die nicht nur die Architektur von Qualitätsbeurteilungen sichtbar werden lassen, sondern auch weiterführende Vergleiche zwischen verschiedenen Typen von Qualitätssicherungssystemen ermöglichen. Da sich zu solchen übergreifenden Strukturfragen bei Reimer eher nur verstreut etwas findet, seien hier die folgenden vier, u. E. besonders wichtigen Stichworte genannt:

\section{Legitimation der Evaluations-Mandarine}

Wenn Qualitätsmessung als notwendige Vorstufe jeder Qualitätssicherungsanstrengungen so wichtig und potentiell folgenreich ist - vom Reputationsverlust einer Hochschule bis zum Staatsbankrott - so ist offenbar die Frage zentral, wer mit welchem Recht Qualitätsurteile abgibt. Diese Frage mag für unterschiedliche Evaluationsbereiche - die Fachgutachter der DFG als die modernen Mandarine des Wissenschaftssystems, die Ratingagenturen als die Hohepriester globalisierter Finanzmärkte, Transparency International als selbsternannte Wächter eines korruptionsgefährdeten Gemeinwohls - unterschiedlich zu beantworten sein, unausweichlich ist sie allemal.

Das Beispiel des „financial auditing“ durch spezialisierte Ratingagenturen mag hier zur Veranschaulichung ausreichen. Der sog. Ratingmarkt weist eine ausgesprochen oligopolistische Struktur auf. Die Agenturen Moody's Investment Services, Standard and Poors, sowie Fitch Ratings teilen sich den gesamten weltweiten Markt nahezu unter sich auf (im Verhältnis von 40:40:15). ${ }^{8}$ Was verschafft hier die notwendige Legitimation? Der nur bedingt funktionsfähige oligopolistische Markt, das Vertrauen der Kunden oder/und die Zulassung der Agenturen durch die US-amerikanische Börsenaufsicht? Das sind spannende Fragen, die aber hier nicht vertieft werden können.

7 Bruno S. Frey, Evaluitis - eine neue Krankheit, in: Matthies/Simon (Fn. 1), S. 125 ff.

8 Angaben bei Hornbostel (Fn. 2), S. 64. 


\section{Beobachtungsstrukturen als Governancestrukturen}

Wenn wir in einer Welt leben, in der alles beobachtet, gemessen und gewichtet wird, dann bedarf es dazu beobachtender Organisationen, die bestimmte Beobachtungsverfahren anwenden und deren Beobachtungsergebnisse in einen komparativen und kompetitiven politischen Prozess eingespeist werden, in dem diese Ergebnisse über die Zauberformel „best practice“ und „benchmarking“ erhebliche Steuerungsleistungen erreichen. ${ }^{9}$ Man kann insoweit von institutionalisierten Beobachtungs- und Bewertungsstrukturen sprechen: solche Governancestrukturen finden sich etwa - um nur drei Beispiele zu nennen - bei der Qualitätskontrolle von Lebensmitteln, ${ }^{10}$ der ständigen Beobachtung und Bewertung der ökonomischen Performance von Staaten durch die Weltbank ${ }^{11}$ oder der institutionalisierten Beobachtung der Politiken ihrer Mitgliedstaaten durch die Reputationsgemeinschaft der OECD ${ }^{12}$ - das Stichwort Pisa mag hier genügen.

Häufig führen diese Beobachtungen zu Anforderungen, die an die beobachteten Organisationseinheiten gestellt werden, indem ihnen etwa aufgegeben wird, bestimmte organisatorische Vorkehrungen zu treffen, die - sei es als Bausteine ihrer „corporate governance"-Strukturen, sei es als Element ihrer Verwaltungsstruktur - eine qualitätssichernde Funktion erfüllen sollen. Hierzu findet sich eine Menge Anregendes in der Arbeit von Franz Reimer, und zwar in dem Abschnitt mit der Überschrift „Qualität durch Systemanforderungen“" (S. $331 \mathrm{ff}$.). Neben der Auflage an bestimmte Betreiber ihrerseits ein sog. Qualitätsmanagement zu betreiben - nach $\$ 11$, Abs. 2 Nr. 4 HeimG darf ein Heim „nur betrieben werden, wenn der Träger ein Qualitätsmanagement betreibt" - sind dies - wie Reimer sie nennt - Anforderungen an die „Binnenselbstregulierung“; dazu rechnet er in einer überaus einleuchtenden Systematisierung die folgenden Anforderungen:

- Auferlegung von Konzeptpflichten etwa in Form eines „tragfähigen Geschäftsplanes“

- Auferlegung von Organisationspflichten etwa zur Bestellung von Beauftragten

- Auferlegung von Verfahrenspflichten, etwa in Gestalt von Informationseinholungspflichten von Kreditinstituten

9 Siehe dazu Elke Löffler, A Survey on Public Sector Benchmarking Concepts, in: Hermann Hill/Helmut Klages/Elke Löffler (Hrsg.), Quality, Innovation and Measurement in the Public Sector, Frankfurt am Main 1996, S. $137 \mathrm{ff}$.

10 Gunnar Folke Schuppert, Verantwortung, Kompetenz, Kontrolle. Verwaltungswissenschaftliche Überlegungen zur Neuorganisation des Verbraucherschutzes in Deutschland, in: ZLR (Zeitschrift für das gesamte Lebensmittelrecht) Nr. 3/2002, S. $297 \mathrm{ff}$.

11 Siehe dazu Christoph Möllers, Die Governance-Konstellation. Transnationale Beobachtung durch öffentliches Recht, in: Gunnar Folke Schuppert/Michael Zürn (Hrsg.), Governance in einer sich wandelnden Welt, PVS-Sonderheft 41 (2008), S. 238 ff.

12 Materialreich dazu Martin Schäfer, Die neue Unverbindlichkeit. Wirtschaftspolitische Koordination in Europa, Frankfurt am Main 2005. 
- Auferlegung von Dokumentationspflichten etwa in Form der Aufzeichnung von Arbeitsschritten

- Auferlegung von Zugänglichkeits- und Rechenschaftspflichten etwa zur Errichtung eines Beschwerdemanagements

Es ist naheliegend, von hier einen Bogen zu spannen zu der verwaltungswissenschaftlichen, aber auch verwaltungsrechtswissenschaftlichen Diskussion über Organisation und Verfahren als Steuerungsfaktoren, ${ }^{13}$ um auf diese Weise das wichtige Instrument der Qualitätsgewährleistung durch Systemanforderungen in die allgemeine steuerungswissenschaftliche Diskussion einzubetten.

\section{Zur Problematik von Qualitätsindikatoren}

„Die Frage nach der Messung (bescheidener: Bewertung) von Dienstleistungsqualität zählt daher zu den heikelsten einer juristischen Qualitätslehre überhaupt“. Diesem Satz von Franz Reimer ${ }^{14}$ ist nicht nur uneingeschränkt zuzustimmen, sondern er kann dahin verallgemeinert werden, dass die Methoden der Qualitätsmessung die Achillesferse jeder Qualitätslehre sind. Dies wird besonders deutlich, wenn es nicht um die Qualität einzelner Produkte geht, sondern von komplexen Beurteilungsgegenständen wie sozialen oder medizinischen Dienstleistungen oder auch von Regierungssystemen oder von Governanceregimen. Wenn es dem Rezensenten erlaubt ist, kurz bei diesem ihm besonders vertrauten Terrain zu bleiben, so sei ein kurzer Seitenblick auf die Frage geworfen, wie man eigentlich Staatsqualität bzw. Governancequalität messen kann.

Was zunächst die Messung von Staatsqualität angeht, so ist dieses Problem aus der Debatte über die sog. Räume begrenzter Staatlichkeit vertraut. ${ }^{15}$ Ob ein ins Auge gefasster Staat zufriedenstellend funktioniert - wie beurteilt man dies? - oder in die Gruppe von „weak states“ oder gar „failing states“ gehört, wird anhand von Indikatoren beurteilt, ${ }^{16}$ die von unterschiedlichen Organisationen entwickelt wurden ${ }^{17}$ und sich überwiegend auf Expertenurteile stützen. Damit ist das Herzstück der ganzen, hier nicht auszubreitenden Debatte die Qualität der Indikatoren: Messungsprobleme sind also - und diese Aussage gilt sicherlich für jede Qualitätslehre - Metho-

13 Näher dazu Gunnar Folke Schuppert, Verwaltungsorganisation und Verwaltungsorganisationsrecht als Steuerungsfaktoren, in: Wolfgang Hoffmann-Riem/Eberhard Schmidt-Aßmann/Andreas Voßkuhle (Hrsg.), Grundlagen des Verwaltungsrechts, Band I, München 2006, $\$ 16$.

14 S. 147.

15 Überblick bei Schuppert (Fn. 4), S. $89 \mathrm{ff}$.

16 Vgl. Ulrich Schneckener, States at Risk - Zur Analyse fragiler Staatlichkeit, in: derselbe (Hrsg.), Fragile Staatlichkeit. „States at Risk“ zwischen Stabilität und Scheitern, Baden-Baden 2006, S. 9 ff.

17 Die bekanntesten dürften die von der Weltbank entwickelten Indikatoren sein; siehe Daniel Kaufmann/ Aart Kraay, Governance Indicators: Where are we? Where Should We Be Going? The World Bank Policy Research Working Paper 4730, Washington D.C., 2007. 
denprobleme. ${ }^{18}$ Bei der Messung von Governancequalität verhält sich dies nicht anders. ${ }^{19}$ Zur Veranschaulichung mag hier auf die nachstehende Grafik verwiesen werden, die wir dem Artikel „Measuring Governance“ in dem soeben erschienenen „Sage Handbook of Governance" entnommen haben. ${ }^{20}$

\section{Diagnostic tools for assessing the quality of democratic governance}

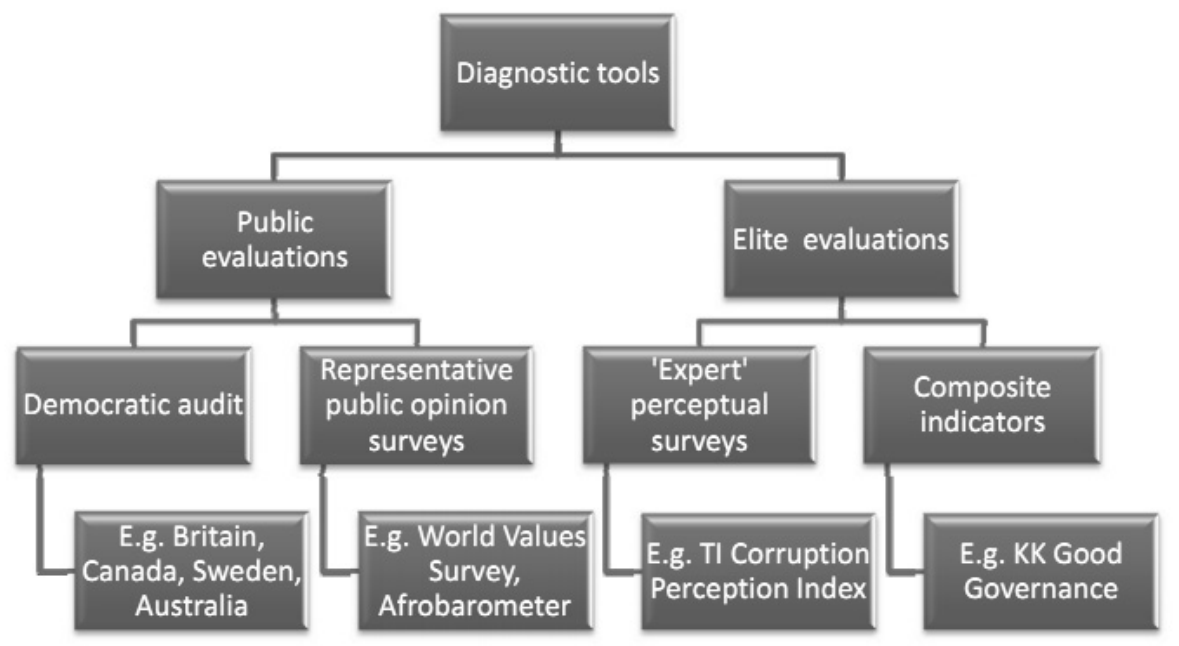

Angesichts der zentralen Bedeutung von Indikatoren und ihrer methodischen Problematik hätte man sich gewünscht, dass die Reimersche Schrift sich hierzu etwas expliziter äußert, als er dies in seiner Passage zur Qualitätsmessung (S. 147 ff.) getan hat.

\section{Qualitätsbewertung als Governance von und durch Wissen}

Das letzte, hier nur kurz anzutippende Stichwort lautet „Governance von und durch Wissen “, ${ }^{21}$ womit der Befund auf den Begriff gebracht werden soll, dass Qualitätsmessungen und vor allem die Entwicklung von Qualitätskriterien überwiegend auf Expertenwissen beruht. Dies gilt nicht etwa nur für die technische Expertise und die von Reimer betonte Technizität von Qualitätskriterien: „Qualität ist drittens tech-

18 Siehe dazu Martin Brusis, Reformfähigkeit messen? Konzeptuelle Überlegungen zu einem Reformfähigkeitsindex für OECD-Staaten, PVS 49 (2008), S. 92 ff.

19 Laura Müller, Governance messen? Eine Bestandsaufnahme quantitativer empirischer Ansätze, in: Sybille de la Rosa/Ulrike Höppner/Matthias Kötter (Hrsg.), Transdisziplinäre Governance-Forschung. Gemeinsam hinter den Staat blicken, Baden-Baden 2008, S. $270 \mathrm{ff}$.

20 Pippa Norris, Measuring Governance. The Sage Handbook of Governance, London 2011, S. $179 \mathrm{ff}$., 182.

21 Siehe dazu die Beiträge in Gunnar Folke Schuppert/Andreas Voßkuble (Hrsg.), Governance von und durch Wissen, Baden-Baden 2008. 
nizistisch. Daher liegen eine Orientierung an fachlichen Standards des jeweils dominanten Sachgebietes und eine Quantifizierung nahe. Tatsächlich kann in der Übernahme wissenschaftlicher Standards eine Delegation von Verantwortung und die Verkennung (oder Bemäntelung) der Entscheidungs- und Bewertungsprobleme liegen. “22

Dies gilt vielmehr auch und wohl noch mehr, wenn es nicht nur um technische Standards geht - wie etwa bei der Frage nach der Beschaffenheit von Steckdosen - sondern um Qualitätsprobleme von Luft, Umwelt oder Gesundheit, Bereichen, in denen häufig mit Grenzwerten gearbeitet wird - wie viel Dioxin verträgt ein Frühstücksei? - mit denen zugleich über die Grenzen des Grundrechts auf Gesundheit entschieden wird.

Eine weitere Achillesferse jeder Qualitätslehre also ist das Problem der Auswahl, der Repräsentativität und der Qualität von Experten, so dass wir es hier mit einem Paradefall von Governance von und durch Wissen zu tun haben.

\section{Der „Zur Rolle des Staates“-Diskurs}

Wenn es um die Rolle des Staates geht, so wird diese Diskussion - und zwar nicht nur in Deutschland - als Diskussion über handlungsleitende und perspektivenerweiternde Staatsbilder geführt. ${ }^{23}$ Diese Staatsbilder - heißen sie Dienstleistungsstaat, Präventionsstaat oder aktivierender Staat - dienen dabei als Rollenmetaphern, ${ }^{24}$ mit denen die angemessene Rollenverteilung zwischen Staat, Markt und Zivilgesellschaft beschrieben werden soll. Was nun den Bereich der Qualitätssicherung im Geflecht zwischen marktlicher und staatlicher Steuerung angeht, so scheint der Typus des Gewährleistungsstaates ein sich aufdrängender Kandidat für das problemangemessene Staatsbild zu sein; dies sieht auch Reimer so, wenn er formuliert: „Qualitätssicherung ist ein, vielleicht gar der Modus der Gemeinwohlgewährleistung im Gewährleistungsstaat" ${ }^{25}$ Ganz in diese Richtung argumentiert auch Margrit Seckelmann, die in ihrem Beitrag im „Handbuch zur Verwaltungsreform“ Akkreditierung und Zertifizierung „als Verfahren der Qualitätssicherung im Gewährleistungsstaat“ behandelt ${ }^{26}$ und dazu Folgendes ausgeführt hat: „Beiden gemeinsam ist eine Verantwortungsteilung von Staat und Gesellschaft bzw. eine Aktivierung gesellschaftlichen selbstregulativen Potentials und seine Einbindung in die Wahrnehmung von

22 S. 148.

23 Zur Verwendungsweise und Funktion von Staatsbildern siehe Andreas Voßkuble, Der „Dienstleistungsstaat“. Über Nutzen und Gefahren von Staatsbildern, in: Der Staat 40 (2001), S. 495 ff.

24 Begriff bei Schuppert (Fn. 4), S. 63.

25 S. 284.

26 Margrit Seckelmann, Akkreditierung und Zertifizierung, in: Bernhard Blanke et al. (Hrsg.), Handbuch zur Verwaltungsreform, 4. Aufl., Wiesbaden 2011, S. $501 \mathrm{ff}$. 
Staatsaufgaben, für die der Staat zumindest die Gewährleistungs- (und damit auch die Überwachungs-) verantwortung trägt“ .27

Angesichts dieses Befundes, dass Qualitätssicherungsaufgabe und Gewährleistungsstaat ein offenbar perfektes Paar abgeben, ist der Leser - zumindest aber der Rezensent - etwas überrascht, dass Franz Reimer nicht diese Paarbeziehung näher strukturiert, sondern einen anderen Staatstyp die Bühne betreten lässt, nämlich den von ihm so genannten Meliorationsstaat, dessen deutsche Bezeichnung - „Verbesserungsstaat" - keine große Karriere erwarten lässt. Reimer sieht diesen Meliorationsstaat vor allem in dem europarechtlichen Optimierungsdenken verankert; da der Rezensent - wie wohl auch manch anderer Leser - diesem Staatstypus noch nicht begegnet ist, sollten wir einen Blick darauf werfen, wie Reimer ihn präsentiert: „Das permanente Verbesserungsstreben hat seine Heimat nicht nur in betriebswirtschaftlichen Programmatiken, sondern auch im europapolitischen Diskurs und im europarechtlichen Sprachgebrauch... Der Staat ist danach nicht nur Vorsorgestaat und »Förderungsstaat «, sondern auch »Verbesserungsstaat «. Nicht weniger widmet sich die Europäische Gemeinschaft, nun die Union - auf dem hier betrachteten Gebiet privater Dienstleistungen - sowohl durch Induzierung politischer Prozesse als auch durch Sekundärrecht - der Hebung der gesamtgesellschaftlichen Wohlfahrt, mit anderen Worten: der "guten Policey «. Gelegentlich tut sie dies in nachgerade naivem Regulierungsglauben. Neben liberalisierendes und deregulierendes Handeln der Gemeinschaftsorgane tritt - möglicherweise aufgrund des permanenten Legitimationsdrucks und der schrittweisen Verschiebung von input- zu output-Legitimation - zunehmend ein gesellschaftsveredelndes, meliorierendes Handeln. "28

Wir können nicht so recht erkennen, wie der Begriff des Meliorationsstaates unsere analytische Sehschärfe im Bereich des Problems der Qualitätssicherung zu erhöhen vermag; vielmehr möchten wir - was uns hoffentlich nicht als Altersstarrsinn zugerechnet wird - am Konzept des Gewährleistungsstaates festhalten, weil nicht nur der ihm zugrunde liegende Gedanke einer Verantwortungsteilung gut passt, er vielmehr auch für das wichtige Problem des Zusammenspiels von selbstregulativer Eigenkontrolle und staatlicher Fremdkontrolle fruchtbar gemacht werden kann.

\section{Der Ökonomisierungs-Diskurs}

Vollkommen zu Recht hat Reimer die Heimstatt des Qualitätsdenkens in den Wirtschaftswissenschaften ausgemacht und der Qualitätssicherung in den Wirtschaftswissenschaften einen eigenen, sehr informativen Paragraphen gewidmet. ${ }^{29}$ Unter dem Gesichtspunkt der Entwicklung einer „Allgemeinen Qualitätslehre“ ist nun der

27 Seckelmann (Fn. 26), S. $506 \mathrm{f}$.

28 S. $291 \mathrm{ff}$.

29 S. $115 \mathrm{ff}$. 
Befund interessant, dass das Denken in Management-Kategorien - Stichwort: Qualitätsmanagement - längst den angestammten Platz von durch Private erbrachten Dienstleistungen verlassen, sondern den gesamten öffentlichen Sektor erfasst hat, ein Vorgang, der unter der Überschrift „Ökonomisierung des öffentlichen Sektors“ intensiv diskutiert worden ist. ${ }^{30}$ Das trojanische Pferd, mittels dessen dieser Kolonisierungsvorgang bewirkt worden ist, hört auf den Namen „New Public Management“: „Öffentliche Verwaltungen an ibren Leistungen zu messen und sie auf Verbesserung dieser Leistungen steuernd auszurichten - das ist das zentrale Credo der New Public Management-Bewegung. “31

Ein - wie die jüngste Vokabel heißt - „Performance Management“ beansprucht also Geltung für jede Bereitstellung - sei es privatrechtlich oder öffentlich-rechtlich erbrachter Dienstleistungen und die Bereitstellung öffentlicher Güter, ein Befund, den Frank Nullmeier bündig wie folgt zusammengefasst hat: 32

„Der Bereitstellungsprozess eines Gutes, einer Dienstleistung oder auch die Erfüllung einer komplexen öffentlichen Aufgabe kann mit den aus der Kybernetik bzw. Systemtheorie stammenden Begriffen Input (für den Ressourcen-, Mittel oder Faktoreinsatz), Output (für das Faktorergebnis, den Ertrag oder die erbrachte Leistung) und Outcome (für die mit der Leistung erzielten Wirkungen) erfasst werden. Unter Performance einer öffentlichen Verwaltung werden sowohl die Leistungen im Sinne des Outputs als auch die weiteren Wirkungen in der Gesellschaft im Sinne des Outcomes bezeichnet. Unter Performance-Management kann man daher eine Kombination aus Output-(Leistungs-) und Outcome (Wirkungs-)Steuerung verstehen, unter Performance Measurement die systematische Erhebung und Bewertung der Leistungen und Wirkungen."

Nach unserem Eindruck - den auch Reimer zu teilen scheint - ist der Zenit der Dominanz ökonomischer Imperative überschritten, so dass es an der Zeit wäre, sich auf die spezifische Legitimation und Funktionslogik des öffentlichen Sektors zurückzubesinnen. In diesem Zusammenhang wäre zu überlegen, ob es nicht nur eines juristischen Qualitätsbegriffs bedarf - wozu sich bei Reimer weiterführende Überlegungen finden ${ }^{33}$ - sondern ob es möglich wäre, einen Qualitätsbegriff für die Bereitstellung öffentlicher Güter zu entwickeln, der den Adressaten und Abnehmer nicht primär als Konsumenten oder Kunden im Blick hat, sondern als Bürger, der in unserem Verfassungsstaat nicht nur eine ökonomische Größe ist, sondern mitbestimmender

30 Siehe dazu die Beiträge in Jens Harms/Christoph Reichard (Hrsg.), Die Ökonomisierung des öffentlichen Sektors, Baden-Baden 2003.

31 Frank Nullmeier, Output-Steuerung und Performance Measurement, in: Handbuch zur Verwaltungsreform (Fn. 26), S. 465.

32 S. 465.

33 S. $135 \mathrm{ff}$. 
und grundrechtsbewährter Governance-Akteur. Aber dieser reizvolle Gedanke kann hier nicht weiter fortgesponnen werden.

\section{Der „Das Gesetz bekommt Gesellschaft“-Diskurs}

Die juristische Welt wäre in bester Ordnung, würden in dem im Entstehen begriffenen Qualitätssicherungsrecht die entscheidenden Fragen - Träger, Verfahren, Organisation und Qualitätskriterien - gesetzlich oder untergesetzlich, jedenfalls normativ verbindlich geregelt. Nun verhält es sich aber unstreitig so, dass viele für die Qualitätsmessung und Qualitätssicherung relevanten Regelungen sich in anderen Regelwerken als dem von Gesetz, Verordnung und Satzung erfassten Bereich finden, nämlich in Standards und in Codes of Conduct. Mit anderen Worten: das Gesetz bekommt Gesellschaft. In der Welt der Standards ${ }^{34}$ spielen Qualitätsstandards eine überaus wichtige Rolle ${ }^{35}$ und auch die sog. Codes of Conduct, die wie Pilze aus dem Boden schießen - insbesondere nach Skandalen und von NGOs angeprangerten Missständen - formulieren häufig Verhaltens- und Qualitätsstandards. ${ }^{36}$

Diesem Befund verschließt sich die Schrift von Franz Reimer nicht, eher im Gegenteil. In einem äußerst lesenswerten Abschnitt behandelt Reimer Erscheinungsformen und Funktionen privater Regelwerke ${ }^{37}$ und an anderer Stelle ebenso informativ wie kenntnisreich die in von ihm sog. Qualitätscharten formulierten Qualitätsanforderungen. ${ }^{38}$ Was die Qualitätsfunktion privater Regelwerke angeht - auf die wir uns hier beschränken wollen - so unterscheidet er vier solcher Funktionen, nämlich

- die Aufschlüsselungsfunktion. Damit ist gemeint, dass solche Regelungen Anforderungen an die betreffenden Dienstleistungen spezifizieren und die komplexen Dienstleistungen dadurch näher aufschlüsseln.

- die Informationsfunktion. Private Regelwerke transportieren gesellschaftliches Wissen und entfalten aufgrund dessen eine gewisse Eigendynamik, obwohl - wie Reimer immer wieder betont - „fachliche Regeln in der Rechtsordnung Geltung nur aufgrund (kontrollierender) Rezeption beanspruchen " können. ${ }^{39}$

- die Dynamisierungsfunktion. Damit ist gemeint, dass der Staat mittels der Technik „wohlwollender“ Rezeption ${ }^{40}$ an der raschen Entwicklung gesellschaftlichen Wis-

34 Nahezu alles, was man von der Welt der Standards wissen muss, findet sich bei Nils Brunsson/Bengt Jacobsson (Hrsg.), A World of Standards, Oxford, 2000.

35 Nils Brunsson/Bengt Jacobsson, The Contemporary Axpansion of Standardization, in: dieselben (Fn. 34), S. $3 \mathrm{ff}$.

36 Informativer Überblick bei Rhys Jenkins, Corporate Codes of Conduct. Self-Regulation in a Global Economy. United Nations Research Institute for Social Development, Programme Paper No. 2, Genf 2001.

37 \$ 8, S. $203 \mathrm{ff}$.

38 S. $304 \mathrm{ff}$.

39 S. 215.

40 Begriff von uns. 
sens partizipieren will, ohne die stabile gesetzliche Verhaltensorientierung aufgeben zu müssen.

- die Internationalisierungsfunktion. Diese sei deshalb wichtig, weil die Einbeziehung privater Regeln und Standards in die Rechtsordnung die dynamische Rezeption internationaler Entwicklungen erleichtere.

Wie die knappen Erläuterungen zu den genannten Funktionen ausreichend belegen, geht es bei Reimer immer um die Inkorporation solcher nicht-staatlicher Regelwerke in die geltende Rechtsordnung, und zwar durch das, was in der Juristensprache Rezeption heißt, mittels derer nicht-staatliche Regelungen in den Adelsstand verbindlichen Rechts erhoben werden. Dies heißt aber, auf halbem Wege stehen zu bleiben und aus dem Befund einer Regelungspluralität nicht die erforderlichen Konsequenzen zu ziehen. Uns erscheint es aus rechtssoziologischer Perspektive unvermeidlich und an der Zeit zu sein, die Fixierung auf das Gesetz und die Gesetzgebungslehre zu überwinden und zur Kenntnis zu nehmen, dass die Welt der Verhaltensregeln "the world of rules" - nicht nur aus normativ verbindlichen Rechtsregeln besteht, sondern eine Welt pluraler Regelungsarten existiert, die als Gesamtphänomen in den Blick genommen werden muss; wir haben mit der Idee einer Regelungswissenschaft dazu einen ersten Schritt zu tun versucht. ${ }^{41}$

Für eine zu entwickelnde Qualitätslehre bedeutet dies, dass wir es mit einer Pluralität von Qualitätsregelungen zu tun haben, die insgesamt - wie beim Verhältnis von verbindlichem Recht und „soft law“ im Mehrebenensystem ${ }^{42}$ - einen Regelungsverbund konstituieren, den es hinsichtlich seiner Gesamtarchitektur zu analysieren gilt. Ein besonderes Augenmerk hätte dabei den Möglichkeiten eines Zusammenspiels der verschiedenen Regelungsarten in einem solchen Regelungsverbund zu gelten, etwa durch die in den verschiedensten Facetten vorkommende Technik der „regulierten Selbstregulierung ". ${ }^{43}$ Aber das kann hier leider nicht vertieft werden.

\section{Der Governance-Diskurs}

Der Governance-Diskurs gehört zu den intensivsten sozialwissenschaftlichen Diskursen überhaupt. ${ }^{44}$ Aber nicht deshalb soll er hier eingeführt werden, sondern weil sein Analysepotential auch für das Thema der Qualitätssicherung fruchtbar gemacht werden kann: wir denken dabei vor allem an den von Hans-Heinrich Trute et al. in die verwaltungsrechtliche Diskussion eingebrachten Begriff des Governancere-

41 Governance und Rechtsetzung. Grundfragen einer modernen Regelungswissenschaft, Baden-Baden 2011.

42 Vgl. dazu die interessante Schrift von Matthias Knauff, Der Regelungsverbund: Recht und Soft Law im Mehrebenensystem, Tübingen 2010.

43 Weiterführend dazu nunmehr Peter Collin, „Gesellschaftliche Selbstregulierung“ und „Regulierte Selbstregulierung “ - ertragreiche Analysekategorie für eine (rechts-)historische Perspektive? (i.E.)

44 Siehe dazu den Überblick bei Gunnar Folke Schuppert, Alles Governance oder was? - Zur Leistungsfähigkeit des Governance-Konzepts, Baden-Baden 2011. 
gimes. ${ }^{45}$ Denn Qualitätssicherungssysteme sind nichts anderes als Governanceregime, eine These, die wir in zwei Schritten begründen wollen.

In einem ersten Schritt wollen wir einen Blick auf einen typischen Fall von Qualitätssicherungssystemen werfen, nämlich auf Evaluationsregime. Stefan Hornbostel, einer der besten Kenner dieser Materie, hat in seinem Überblick über verschiedene Evaluationsregime - sein Lieblingsbeispiel ist die Evaluierungsinstitution der Inquisition - Evaluationsregime - er denkt dabei vor allem an den Bereich der Wissenschaft - wie folgt beschrieben: ${ }^{46}$

„Evaluation - die Bewertung und Prüfung von Sachverhalten, Leistungen oder auch von Konformitäten - ist sicherlich nicht neu. Das gilt insbesondere für die Evaluation von Wissen und Erkenntnisansprüchen. Es lohnt daher, exemplarisch die Evaluationsregime aus zwei Wissenskulturen im Hinblick auf ibre Organisation und Trägerschaft, auf Kriterien und Verfahren, auf machtstabilisierende oder destabilisierende Funktionen und auf mögliche Sanktionen bzw. soziale Folgen genauer zu betrachten."

Es geht also bei Evaluationsregimen um ein bestimmtes Arrangement von Trägerschaft - wer evaluiert? -, von Organisation und Verfahren, von Evaluierungskriterien und dies alles in den Blick genommen unter Berücksichtigung machtpolitischer und sozialer Folgen.

In einem zweiten Schritt wollen wir dem die Definition von Governance-Regimen durch Trute et al. gegenüberstellen: Governanceregime sind zu verstehen „als... aufgabenbezogene institutionelle Arrangements“, wobei das Governanceregime nicht nur aus rechtlichen, sondern auch aus nichtrechtlich verbindlichen, zum Beispiel sozialen Koordinationsmechanismen bestehen kann. ${ }^{47}$

Wenn wir beides zusammenführen, dann können wir Qualitätssicherungssysteme definieren als aufgabenbezogene institutionelle Arrangements, deren Elemente nicht nur aus normativ verbindlichen rechtlichen Vorgaben, sondern auch aus nicht-staatlichen Regelungswerken bestehen können. Wenn wir so vorgehen, sind wir nicht nur problemlos anschlussfähig an das, was wir oben als Regelungsverbund vorgestellt haben, sondern finden auch einen leichten analytischen Zugang zu einer Aufgabenund Verantwortungsteilung zwischen Markt, Staat und Zivilgesellschaft wie etwa bei Akkreditierungs- und Zertifizierungsverfahren sowie zu dem erwähnten Zusammenspiel von gesellschaftlicher Selbstregulierung und staatlicher Regulierung in Gestalt einer „regulierten Selbstregulierung“.

45 Hans-Heinrich Trute/Doris Kühlers/Arne Pilniok, Governance als verwaltungsrechtswissenschaftliches Analysekonzept, in: Gunnar Folke Schuppert/Michael Zürn (Hrsg.), Governance in einer sich wandelnden Welt, PVS-Sonderheft 41/2008, Wiesbaden, S. $173 \mathrm{ff}$.

46 Hornbostel (Fn. 2), S. 59.

47 Trute/Kühlers/Pilniok (Fn. 45), S. 178. 


\section{Drei Beispiele für qualitätssichernde Governanceregime}

Im Folgenden wollen wir uns drei qualitätssichernde Governanceregime etwas näher ansehen und dabei zugleich herauszufinden versuchen, ob bei ihnen die obige Charakterisierung von Governanceregimen als aufgabenbezogenen institutionellen $\mathrm{Ar}$ rangements mit einem Ineinandergreifen von verschiedenen qualitätsbezogenen Regelungsarten wirklich zutrifft.

\section{Selbststeuerung durch peer review}

Einen wohl eher atypischen Fall von peer review hat sich Franz Reimer genauer angesehen, nämlich die Qualitätssicherung durch peer review im Bereich des Wirtschaftsprüfungsrechts. ${ }^{48}$ Es dürfte sich hier um einen der wenigen Fälle handeln, in denen diese Strategie der Qualitätssicherung durch peer review-Kontrolle gesetzlich geregelt ist; die entsprechende Vorschrift des Wirtschaftsprüferrechts ( $\$ 55 \mathrm{~b}$ WiPrO) lautet wie folgt: „Der Wirtschaftsprüfer oder die Wirtschaftsprüferin hat die Regelungen, die zur Einhaltung der Berufspflichten erforderlich sind, zu schaffen sowie ihre Anwendung zu überwachen und durchzusetzen (Qualitätssicherungssystem). Das Qualitätssicherungssystem ist zu dokumentieren.“

Interessant ist nun aber, in welchen Regelungsregimen sich nun die Maßstäbe für dieses Qualitätssicherungssystem finden; im Gesetz nämlich nicht, sondern - wie Reimer zusammengestellt hat - in privaten Regelungswerken, sei es des Berufsstandes, sei es von privaten Standard Setting Committees: ${ }^{49}$

„In Bezug genommen werden damit zunächst die Allgemeinen Berufspflichten wie Unabhängigkeit, Gewissenhaftigkeit, Verschwiegenheit und Eigenverantwortlichkeit ( $\$ 43$ Abs. 1 S. $1 \mathrm{WiPrO}$ ), berufswürdiges Verhalten (\$ 43 Abs. 2 WiPrO), Fortbildung ( $\$ 43$ Abs. 2 S. 4 WiPrO), sowie die Besonderen Berufspflichten (\$ 57 Abs. 4 Nr.2f., 5 i.V.m. der Berufssatzung der Wirtschaftsprüferkammer). Die Berufssatzung statuiert in $\$ 4$ Abs. 1 eine Bindung an "fachliche Regeln «; diese ergänzen die gesetzlichen Regeln und liegen vor allem in den Grundsätzen ordnungsmäßiger Buchführung i.S.v. $\$ 238$ Abs. 1 S. 1 HGB, den Verlautbarungen des Deutschen Rechnungslegungs Standards Committee e.V. sowie den Prüfungsstandards, -hinweisen, Stellungnabmen zur Rechnungslegung und Rechnungslegungshinweisen des Instituts der Wirtschafsprüfer in Deutschland e.V. (IDW).“

So interessant dieser Fall eines gesetzlich vorgesehenen peer review-Verfahrens auch ist, so wenig scheinen uns in ihm die typischen Probleme von peer review deutlich zu werden. Deshalb wollen wir ergänzend einen Blick auf das im Wissenschaftsbe-

49 S. 367; zur Rolle solcher nicht-staatlicher Standardsetter siehe Gunnar Folke Schuppert, Governance und Rechtsetzung (Fn. 41), S. 217 f. 
reich immer mehr an Bedeutung gewinnende peer review-System werfen, das gänzlich gesetzesfrei funktioniert, dessen verhaltenssteuernde Normen durchweg informaler Natur sind und dessen Funktionslogik mit dem Begriff von „governance by reputation" ${ }^{50}$ gekennzeichnet werden kann: Veröffentlichungen in peer review-Journals vermitteln Reputation und bisher angesammelte Reputation ist die Voraussetzung zur Bestellung als peer.

Was zunächst die Funktion des peer review-Verfahrens angeht, so hat ein wirklich exzellenter Kenner des Wissenschaftssystems - Friedhelm Neidhardt - dazu Bedenkenswertes gesagt; er spricht nicht nur von der uns allen bekannten Selektionsfunktion, sondern auch von einer Konstruktionsfunktion, weil peer review ein wesentliches Element für die Konstituierung einer „scientific community“ darstellt; dazu heißt es bei ihm wie folgt: ${ }^{51}$

„Mit Peer Review geht es um den Versuch, Qualitätskontrollen in der Wissenschaft professionell und konstruktiv zu gestalten. Zwei Funktionen von Peer Review spielen dabei eine Rolle: Es geht einerseits um Selektionsfunktionen, nämlich um die Auswahl von Personen, Projekten und Texten für den Zuschlag knapper symbolischer und materieller Ressourcen. Da entsprechende Entscheidungen aber in der Regel nicht im Sinne schlichter Ja/Nein-Muster und in einem Zug zu treffen sind, werden sie häufig konditioniert und prozessualisiert, nämlich an Auflagen gebunden, deren Erfüllung oder Nichterfüllung über den Ausgang der Evaluation entscheidend sind. Ein beträchtlicher Teil von Forschungsmitteln und Publikationschancen werden erst nach Korrekturen von Anträgen und Manuskripten vergeben, zu denen Beanstandungen und Empfehlungen der Gutachter Anlass gaben. Neben die Selektionsfunktionen von Peer Review treten also auch Konstruktionsfunktionen. Die Peers greifen als Gutachter sowohl probibitiv als auch produktiv in den Wissenschaftsprozess ein, um die von ihnen wahrgenommenen Fachstandards durchzusetzen. Erst über Peer Review konstituieren sich insoweit Wissenschaftsdisziplinen und Forschungsfelder als Scientific Communities.

Erhielt Peer Review also in zunehmendem Maße eine konstitutive Rolle für die Wissenschaften, stellt sich umso mehr die Frage, ob es als professionelles Steuerungsprinzip tatsächlich hinreichend funktioniert. Unter welchen Bedingungen reicht seine eigene Qualität aus, um die Qualität von Personen, Projekten und Texten wissenschaftsgerecht einschätzen und konditionieren zu können?"

$50 \mathrm{Zu}$ „governance by reputation“ und zu Berufsgemeinschaften als Reputationsgemeinschaften siehe Schuppert, Law without the State?, erscheint in: Ursula Lehmkuhl/Thomas Risse (Hrsg.), Governance without the State (i. E.).

51 Friedhelm Neidhardt, Selbststeuerung der Wissenschaft: Peer Review, in: Dagmar Simon/Andreas Knie/Stefan Hornbostel (Hrg.), Handbuch Wissenschaftspolitik, Wiesbaden 2010, S. $281 \mathrm{f}$. 
$\mathrm{Zu}$ diesen von Neidhardt genannten Funktionsbedingungen gehört zuvörderst die Gewinnung kompetenter Gutachter; diese unmittelbar einleuchtende Voraussetzung wirft aber unter dem Gesichtspunkt der Funktionsfähigkeit des Review-Systems Probleme auf, da Kompetenz- und Befangenheitsgefahr tendenziell Hand in Hand gehen: ${ }^{52}$

„Die Qualität sowohl übereinstimmender als auch voneinander abweichender Urteile hängt wesentlich von der fachlichen Kompetenz der Gutachter ab. Nicht einfach ist es allerdings, kompetente Gutachter zu rekrutieren, auf deren Urteil in den anstehenden Fällen auch vertraut werden kann. Immer tritt bei Gutachterrekrutierungen nämlich ein Problem dadurch auf, dass es eine offenkundig inverse Beziehung zwischen Expertise und Befangenheit gibt. Die Bemühung um Kompetenzsteigerung führt zu Gutachtern mit immer größerer Nähe sowohl zu den Forschungsthemen als auch zu deren Bearbeitern. Große Nähe bringt aber mit hoher Wahrscheinlichkeit Interessen und Affekte ins Spiel, die einen Bewertungsbias auslösen. Um diesen zu begrenzen, gibt es einen Bedarf an mittleren Distanzen zwischen den Experten und den Gegenständen, Gruppen und Personen, die sie zu benoten haben. Gutachter müssen der Sache nahe genug und den Kollegen, um die es geht, fern genug sein, damit man ibre unbefangene Urteilskraft erwarten kann."

Als Mittel der Distanzsicherung bietet sich das Anonymitätsprinzip geradezu an; ${ }^{53}$ die politische Vierteljahresschrift etwa, aber auch andere Zeitschriften, arbeiten mit einem „double blind“-Verfahren, in dem weder der Autor des Manuskripts, noch die Gutachter bekannt sind und diese letzteren auch untereinander nicht wissen, wer der Zweitgutachter ist. Nach unserer Kenntnis funktioniert dieses Verfahren gut, und es wird interessant sein, zu beobachten, ob es sich auch im Bereich juristischer Zeitschriften durchsetzen wird.

\section{Akkreditierung im Hochschulbereich oder Steuerung durch Organisation}

Das um sich greifende Akkreditierungswesen ist von komplexer Bauart; am besten scheint uns diese getroffen durch den Titel der 2008 erschienenen Schrift von Karin Bieback, der wie folgt lautet: „Zertifizierung und Akkreditierung. Das Zusammenwirken staatlicher und nicht-staatlicher Akteure in gestuften Prüfsystemen “ ${ }^{54} \mathrm{Ge}$ nau darum geht es: um ein Zusammenspiel nicht-staatlicher und staatlicher Akteure in einem in sich gestuften Governanceregime.

Bevor dieses Governanceregime in aller Kürze skizziert wird, ist an den europäischen Regelungskontext zu erinnern, denn ohne diesen ist die Ausbreitung der Akkredi- 
tierungsagenturen nicht zu verstehen; diesen europäischen Hintergrund erklärt uns Margrit Seckelmann in geraffter Form wie folgt: ${ }^{55}$

„Der Begriff der Akkreditierung gewann durch den Prozess der europäischen Einigung Konjunktur: In der Bologna-Erklärung (1999), ... verpflichteten sich die Signatar-Staaten im Rabmen bildungspolitischer Absichtserklärungen zur Schaffung eines einheitlichen "Europäischen Hochschulraums " innerhalb der ersten Dekade des dritten Jahrtausends. ... Hierzu gehört unter anderem die Errichtung eines Systems leicht verständlicher und aufgrund eines Diplomzusatzes (»Diploma Supplement «) vergleichbarer Hochschulabschlïsse .... Daneben tritt die Etablierung eines Leistungspunktesystems, das in Fortführung des 1989 im Rahmen des ERASMUS-Programms eingeführten European Credit Transfer Systems (ECTS) für eine Anerkennung von Studienmodulen auch vor dem jeweiligen Abschluss sorgen und eine größtmögliche Mobilität der Studierenden sorgen soll. Schließlich wird die Entwicklung einer staatenübergreifenden Qualitätssicherung durch Etablierung vergleichbarer Kriterien und Methoden angestrebt."

Innerhalb der Akkreditierung im Bereich des Hochschulwesens sind zwei Arten zu unterscheiden, die „institutionelle Akkreditierung“ und die „Programmakkreditierung“ ${ }^{56}$ Während die institutionelle Akkreditierung dazu dient, „Hochschulen in nicht-staatlicher Trägerschaft umfassend im Hinblick auf die Einhaltung von wissenschaftlichen Qualitätsmaßstäben in Lehre und Forschung zu prüfen “, 57 geht es bei der Programmakkreditierung um die Evaluierung von Studiengängen.

Diese Programmakkreditierung erfolgt in einem gestuften Organisationsregime. Die eigentliche Akkreditierung erfolgt durch sog. Akkreditierungsagenturen, die aber ihrerseits einer Akkreditierung bedürfen, die durch den sog. Akkreditierungsrat erfolgt, dessen Konstruktion Margrit Seckelmann wie folgt beschreibt: ${ }^{58}$

„Der Akkreditierungsrat mit Sitz in Bonn hat die Rechtsform einer rechtsfäbigen (nordrhein-westfälischen) Stiftung des öffentlichen Rechts (»Stiftung zur Akkreditierung von Studiengängen in Deutschland ", als solche eingerichtet durch das Gesetz zur Errichtung einer Stiftung »Stiftung zur Akkreditierung von Studiengängen in Deutschland « vom 15.2.2005.) Er verfügt über 18 Mitglieder, die für vier Jahre bestellt werden. Der Akkreditierungsrat legt seiner Arbeit derzeit die von ihm am 8. Dezember 2009 beschlossenen »Regeln des Akkreditierungsrates für die Akkreditierung von Studiengängen und für die

55 Seckelmann (Fn. 26), S. $502 \mathrm{f}$.

56 Martina Röbbecke, Akkreditierung, in: Dagmar Simon et al. (Fn. 51), S. $334 \mathrm{ff.}$

57 Röbbecke, S. 334.

58 Senckelmann (Fn. 26), S. 504. 
Systemakkreditierung "zugrunde. Nach diesen wird die Akkreditierung für sieben Jahre erteilt, danach hat sich der akkreditierte Studiengang reakkreditieren zu lassen. Der Akkreditierungsrat lässt sich in siebenjährigen Abständen auch selbst evaluieren."

Wie man sich den Gang des Verfahrens der Akkreditierung vorzustellen hat, schildert Martina Röbbecke wie folgt: ${ }^{59}$

„Die Agentur stellt eine Gutachtergruppe zusammen, die sich in der Regel aus drei Professorinnen und Professoren, einem Vertreter der Berufspraxis und einem/einer Studierenden zusammensetzt. Diese Gutachtergruppe besucht die beantragende Hochschule und führt vor Ort verschiedene Gespräche mit der Leitung der Hochschule, den Lehrenden und den Vertretern der Studierenden. An diesen Gesprächen nimmt ebenfalls der oder die zuständige Mitarbeiter/in der Akkreditierungsagentur teil. Anschließend wird auf der Grundlage der eingereichten Unterlagen und der Gesprächsergebnisse ein Bewertungsbericht verfasst. Dabei können sich die Mitglieder der Gutachtergruppe insbesondere auf die inhaltlich-qualitativen Aspekte konzentrieren, während die Akkreditierungsagentur vor allem die Einhaltung der formalen Vorgaben kontrolliert. Dazu gehören die vom Akkreditierungsrat vorgegebenen »Kriterien für die Akkreditierung von Studiengängen " und die "Allgemeinen Regeln zur Durchfübrung von Verfahren zur Akkreditierung und Reakkreditierung von Studiengängen «.

Der Bewertungsbericht wird der Akkreditierungskommission der zuständigen Agentur zugeleitet, und diese beschließt nach einem festgelegten Entscheidungsreglement die Akkreditierung des Studiengangs, eine Akkreditierung mit Auflagen oder eine Ablehnung der Akkreditierung."

\section{Qualitätssicherung durch Contract Governance}

Wenn man das Thema der Qualitätssicherung und eines Qualitätssicherungsrechts umfassend erörtern will, wird man nicht umhin können, nach dem Beitrag des Vertragsrechts zur Qualitätssicherung zu fragen, zumal dieses Problem das tägliche Brot jeder auf Leistungsaustausch zielenden Vertragspraxis sein dürfte. Wie eine den Qualitätsanforderungen der Parteien entsprechende Leistungserbringung vertraglich gesichert werden kann, ist aber nicht nur Gegenstand zivilrechtlicher Leistungsvereinbarungen, sondern auch von Verträgen der öffentlichen Verwaltung, sei es mit ihren privatrechtlich organisierten Trabanten, sei es mit privaten Dritten. In der New 
Public Management-Theorie nennt man solche Verträge auch Leistungsaufträge, die sich wie folgt charakterisieren lassen: ${ }^{60}$

„Wie der Name sagt, stehen im Zentrum des Leistungsauftrags die zu erbringenden Leistungen. Für diese werden mittels Indikatoren messbare Wirkungsund-Leistungsziele definiert. Die für die Leistungserstellung notwendigen Ressourcen werden idealerweise in Form eines Globalbudgets bereitgestellt und sollen Resultat der Leistungsdiskussion sein und nicht eigentliche Steuerungsgröße. Damit trägt der Leistungsauftrag zum Ziel der wirkungsorientierten Verwaltungsführung bei, welche zu einer effektiveren aber auch effizienteren Bereitstellung öffentlicher Leistungen führen soll.“

Wie in solche und andere Verträge ein funktionsfähiges Qualitätssicherungssystem eingebaut werden kann, ist Gegenstand des Kontraktmanagements oder - um es mit dem neuesten Zweig der Governance-Forschung zu benennen - von Contract Governance, ${ }^{61}$ also von Governance durch und mit den Mitteln des Vertragsrechts. ${ }^{62}$

Zum Abschluss unserer Überlegungen zur Habilitationsschrift von Franz Reimer möchten wir dafür ein konkretes Beispiel geben, das wir selbst als äußerst lehrreich empfunden haben.

Gemäß $\mathbb{9}$ des Gesetzes über den Ausbau der Schienenwege des Bundes (Bundesschienenwegeausbaugesetz) bedarf die Durchführung der in den Bedarfsplänen aufgenommenen Baumaßnahmen sowie deren Finanzierung einer Vereinbarung zwischen den Eisenbahnen des Bundes und denjenigen Gebietskörperschaften, die den Bau oder Ausbau ganz oder teilweise finanzieren. Nachdem in Konkretisierung dieser gesetzlichen Vorgabe eine Reihe von Einzelvereinbarungen geschlossen worden waren, wurde im Januar 2009 eine umfassende vertragliche Vereinbarung auf den Weg gebracht, und zwar in Gestalt der „Leistungs- und Finanzierungsvereinbarung LuFV - zwischen der Bundesrepublik Deutschland auf der einen Seite und der DB Netz AG, der DB Station und Service AG, der DB Energie GmbH und der Deutschen Bahn AG - den Eisenbahninfrastrukturunternehmen (EIU) des Bundes - auf der anderen Seite. In der Präambel zu diesem Vertragswerk heißt es auszugsweise wie folgt:

„Im Rahmen seines Gewährleistungsauftrags nach Art.87 e Abs. 4 GG stellt der Bund sicher, dass dem Wobl der Allgemeinheit, insbesondere den Ver-

60 Lukas Summermatter, Leistungsaufträge, in: Blanke et al. (Hrsg.), Handbuch zur Verwaltungsreform, Wiesbaden 2011, S. 485.

61 Karl Riesenhuber/Florian Möslein, Contract Governance - Skizze einer Forschungsperspektive, in: Karl Riesenhuber (Hrsg.), Perspektiven des Europäischen Schuldvertragsrechts, Berlin 2008, S. 4 ff.

62 Gunnar Folke Schuppert, Contract Governance. Verwaltungsrecht und Verwaltungswissenschaft vor neuen Herausforderungen, in: Stefan Grundmann et al. (Hrsg.), Festschrift 200 Jahre Juristische Fakultät der Humboldt-Universität zu Berlin, Berlin 2010, S. 1334 ff. 
kehrsbedürfnissen, beim Erhalt der Schienenwege Rechnung getragen wird. Zur Erfüllung des Gewährleistungsauftrags leistet der Bund nach näherer Maßgabe dieser Vereinbarung einen jährlichen Infrastrukturbeitrag.

Die Leistung von Infrastrukturbeiträgen des Bundes erfolgt nach Maßgabe des Bundesschienenwegeausbangesetzes (BSchwAG). Während bislang auf Grundlage von Rabmen- und Sammelvereinbarungen eine einzelmaßnabmenbezogene Finanzierung von Ersatzinvestitionen stattfand, soll der Einsatz der Bundesmittel in Zukunft qualitätsorientiert gesteuert werden. Ziel ist es, zu einer höheren Effizienz des Einsatzes der Bundes- sowie der Unternehmensmittel der EIU zu gelangen und damit eine Verbesserung des Infrastrukturzustands zu erreichen."

Es geht also um eine qualitätsorientierte Steuerung durch contract governance; als Instrumente dazu sieht die LuFV unter anderem vor

- Die Verpflichtung der Eisenbahninfrastrukturunternehmen, jährlich einen Infrastrukturzustands- und Entwicklungsbericht abzugeben (\$14)

- die Parteien verpflichten sich $(\mathbb{S} \mathbb{1 3}, 14)$ auf die Festlegung von Qualitätskennzablen zur Beurteilung der Infrastrukturqualität (Begriff in $\$ 13$ ) sowie

- gemäß $\$ 11$ der Verwendung obliegt die Qualitätsprüfung zuvörderst einem Infrastruktur-Wirtschaftsprüfer in Gestalt einer vom Bund beauftragten Wirtschaftsprüfungsgesellschaft.

Was also hier vertraglich installiert wurde, ist ein ausgefeiltes Qualitätssicherungssystem, das automatisch die Frage auslöst, wie sich ein solches internes Kontrollsystem eigentlich zu den rechtlich vorgesehenen externen Kontrollverfahren verhält, etwa zur Wirtschaftlichkeitskontrolle durch den Bundesrechnungshof. ${ }^{63}$ Damit ist ein Zentralproblem der Qualitätssicherung schlechthin angesprochen, nämlich das Verhältnis interner und externer Kontrollsysteme; aber auch diese interessante Frage kann hier nicht vertieft werden.

\section{Zusammenfassende Würdigung}

Legt man an die Schrift von Franz Reimer zur Qualitätssicherung die strengen Maßstäbe von Helmuth Schulze-Fielitz zur Qualität öffentlich-rechtlicher Forschung an, ${ }^{64}$ so braucht der Autor vor einer Qualitätskontrolle keine Bange zu haben, im Gegenteil. Der Verfasser hat ein wichtiges Buch zu einem wichtigen, bisher vernachlässigten Thema geschrieben. Dabei hat er unglaublich viel Material zusammengetragen, das Gesamtproblem in viele Facetten und Einzelaspekte aufgefächert und die

63 Vgl. dazu Gunnar Folke Schuppert/Florian Meinel, Die Finanzkontrolle der Gewährleistungsverwaltung - Am Beispiel der Prüfungsbefugnisse des Bundesrechnungshofs gegenüber Eisenbahninfrastrukturunternehmen, erscheint in: Verwaltungsarchiv Heft 4/2011.

64 Helmuth Schulze-Fielitz, Was macht die Qualität öffentlich-rechtlicher Forschung aus? in: JöR n.F. 50 (2002), S. $1 \mathrm{ff}$. 
Notwendigkeit der Entwicklung eines Qualitätssicherungsrechts, ja einer Qualitätssicherungslehre eindringlich vor Augen geführt. Da der Verfasser sich als Anhänger des Staatstyps des Verbesserungsstaates „geoutet“ hat, wird er Verständnis dafür haben, dass auch ein von der Qualität des Buches überzeugter Rezensent noch Spielräume für Verbesserungen sieht. Wo diese Spielräume bestehen, hat diese kleine Rezensionsabhandlung aufzuzeigen versucht. 